CI 1.34 to 2.54). These OR were higher in the sub-sample of smokers (3.87 and 2.06, respectively). Binary logistic regression analysis also confirmed that $\mathrm{R}$ allele carriers (CT and TT) have a higher risk of $\mathrm{CAD}(\mathrm{OR}=2.07$, CI 1.09 to 2.95). MMP-9 R2790 locus did not show significant differences between patients and controls. But $\mathrm{QQ}$ genotype and $Q$ allele were significant risk factors in the smoker group. $\mathrm{Q}$ allele carriers ( $\mathrm{RQ}$ and $\mathrm{QQ}$ ) were also significantly associated with $\mathrm{CAD}$ risk in the smoker group $(\mathrm{OR}=1.43$, CI 1.13 to 1.2.26). The R6680 locus did not show significant differences between two groups. And the MMP-9 polymorphism may not be useful as a predictor of the severity of coronary atherosclerosis.

Conclusions MMP-9 -1562T allele and TT genotype are significantly associated with CAD patients from the Uighur Population of China (Xinjiang). This association was stronger in smokers, supporting the conclusion that an interaction between MMP-9 activity and smoking augments CAD risk. Further studies with larger sample size are warranted to confirm these associations in different populations.

\section{e0126 STUDY ON ANTI-OXIDATIVE FUNCTION OF FOUR KINDS OF SCHIZANDRAE LIGNANS}

doi:10.1136/hrt.2010.208967.126

Sun Xin, Xie Yu, Chen Jianguang, Wang Qi, Tian Dan, Li Tan. Life Science Center, Beihua University, Beihua, China

Objective To study the anti-oxidative function of schisandrin $\mathrm{A}$ (SinA), schisandrinB (SinB), schisandrolA (SolA) and schisandrin ester A (SesA).

Methods Using the method of the self oxidation method of pyrogallol, Fenton system.

Results The results shown that all of four kinds of schizandrae lignans have the inhibition function to Superoxide anion radical $\left(\mathrm{O}_{2}{ }^{-} \cdot\right)$, SinB had the highest inhibition rate which could arrive at $68.74 \%$; They also had the same inhibition to hydroxyl radical $(\mathrm{OH})$ and $\operatorname{Sin} B$ have the best effect.

Conclusions schisandrin A ( $\operatorname{Sin} A)$, schisandrin B ( $\operatorname{Sin} B)$, schisandrolA (SolA) and schisandrin ester A (SesA) can be used as a natural antioxidation for human cardiovascular disease treatment and preventive health care.

\section{e0127 DETERMINATION OF PULMONARY ARTERY PRESSURE AND CARDIAC OUTPUT IN RAT}

doi:10.1136/hrt.2010.208967.127

Ping Yuan, Rui Zhang, Dong Liu, Qianqian Liu. Shanghai Pulmonary Hospital, Tongji University, Shanghai, China

Objective To establish a method for determination of pulmonary artery pressure and cardiac output in rat.

Methods 20 Sprgue-Dawely rats were randomly assigned into two groups: control group and pulmonary arterial hypertension (PAH) group. Rats in $\mathrm{PAH}$ group were received a single subcutaneous injection of monocrotaline $(60 \mathrm{mg} / \mathrm{kg})$. The hand-made PE-50 catheters were inserted into pulmonary artery via right jugular vein, which we can perform mean pulmonary artery pressure. Similarly, cardiac output was detected through thermodilution method.

Results After 21 days, compared with control group, mean pulmonary artery pressure was significantly increased $(17.4 \pm 1.8 \mathrm{~mm} \mathrm{Hg}$ in control group vs $61.8 \pm 4.3 \mathrm{~mm} \mathrm{Hg}$ in $\mathrm{PAH}$ group, respectively) and cardiac output was significantly decreased $(130 \pm 5.8 \mathrm{ml} / \mathrm{min}$ in control group vs $71 \pm 6.7 \mathrm{ml} / \mathrm{min}$ in $\mathrm{PAH}$ group, respectively) in $\mathrm{PAH}$ group.

Conclusions This method is a simple and direct method to detect pulmonary artery pressure and cardiac output in rat.

\section{e0128 ANGIOTENSIN-(1-7) INHIBITS VASCULAR REMODELLING IN RAT JUGULAR VEIN GRAFTS VIA REDUCED ERK1/2 AND P38 MAPK ACTIVITY}

doi:10.1136/hrt.2010.208967.128

${ }^{1}$ Wu Jingguo, ${ }^{1}$ Liang Yanbing, ${ }^{1}$ Tang Hao, ${ }^{2}$ Tang Anli, ${ }^{1}$ Ma Zhongfu, ${ }^{1}$ Ma Hong. ${ }^{1}$ The First Affiliated Hospital Sun Yat-sen University/Department of General Internal Medicine, Guangzhou, China; ${ }^{2}$ The First Affiliated Hospital Sun Yat-sen University, Department of Cardiology, Guangzhou, China

Objects To evaluate the effects of Ang-(1-7) on vascular remodelling in vein grafts.

Methods a model of autologous jugular vein grafts in rats was established. With this model system, rats ( $n=12$ per group) underwent autologous jugular vein graft transplantation (Ang-(1-7) and control groups), or a sham operation (sham group) in which grafting was not performed. Three days after operation, minipumps were installed for continuous infusion of Ang-(1-7) $(25 \mu \mathrm{g} / \mathrm{kg} / \mathrm{h})$ or normal saline (control and sham groups) through the jugular vein. Results 4 weeks, weight, blood pressure and heart rate were not significantly different between groups. Typical venous-graft hyperplasia, vascular remodelling, ERK1/2 activity, p38 MAPK activity and proliferating cell nuclear antigen (PCNA) and a-smooth muscle actin (a-SMA) expression present in the control group were attenuated by continuous Ang-(1-7) infusion. Tissue angiotensin II expression was increased in the Ang-(1-7) and control groups but was not different between the groups.

Conclusion The results of the present study indicate that exogenous Ang-(1-7) interferes with the vascular remodelling of autologous jugular vein grafts and significantly inhibits vein-graft intimal hyperplasia via inhibition of vascular tissue ERK1/2 and p38 MAPK activation. Thus, exogenous Ang-(1-7) improves the outcome of vein grafting via attenuation of vascular remodelling.

\section{C0129 EFFECT OF TETRANDRINE ON ANOXIA/REOXYGENATION- INDUCED RELEASE OF PROINFLAMMATORY FACTORS IN CULTURED CARDIOCYTE OF NEONATE RATS}

doi:10.1136/hrt.2010.208967.129

Yuqin Wang, Yuqin Wang. The Pla 252 hospital

Objective To investigate the effect of tetrandrine on anoxia/reoxygenation-induced the release of myocardial enzyme $\mathrm{LDH}, \mathrm{CK}$ and proinflammatory factors: TNF- $\alpha$, IL- $1 \beta$, IL- 6 in cultured cardiocytes of neonate rates.

Methods After cardiocytes were cultured in vitro successfully, it were divided into four group: control group (CON), anoxia/reoxygenation group (A/R), tetrandrine group (Tet), simvastatin (Sim) in random. Each group was treated as follow: CON group - not treated anoxia/ reoxygenation, continuous incubated $26 \mathrm{~h}$ under normal circumstance. A/R group- first anoxia incubate carried, cells were incubated on the non- saccharide non- serum culture medium, which saturate by $95 \%$ argon gases $2 \mathrm{~h}$, reoxygenation incubate followed, cells were incubated in normal circumstance $24 \mathrm{~h} .0 .9 \%$ saline were added into culture fluid before the beginning of reoxygenation. Tet group and Sim group -the procedure of anoxia/reoxygenation was same to $\mathrm{A} / \mathrm{R}$ group, the difference of these two groups was they added Tet $(30 \mu \mathrm{mol} / \mathrm{l})$ or $\operatorname{Sim}(10 \mu \mathrm{mol} / \mathrm{l})$ respectively into culture fluid and incubated $60 \mathrm{~min}$ before anoxia beginning. LDH, CK, TNF- $\alpha$, IL-1 $\beta$, IL- 6 were detected after reoxygenation $24 \mathrm{~h}$.

Result The LDH and CK were increased significantly in $\mathrm{A} / \mathrm{R}$, Tet, and Sim groups compared with CON group $(p<0.01)$. The LDH and $\mathrm{CK}$ in Tet and Sim group were lower significant than $\mathrm{A} / \mathrm{R}$ group $(p<0.01) .2$. The proinflammatory factors TNF- $\alpha$, IL-1 $\beta$ and IL-6 were increased significantly in $A / R$, Tet, and Sim groups compared with CON group $(p<0.01)$. And it were lower significant than $A / R$ 